\title{
Safety aspects and rational use of a naproxen + esomeprazole combination in the treatment of rheumatoid disease
}

This article was published in the following Dove Press journal:

Drug, Healthcare and Patient Safety

3 March 201 I

Number of times this article has been viewed

\author{
David N Roberts' \\ Philip B Miner ${ }^{2}$ \\ 'Department of Internal Medicine, \\ Digestive Diseases Section, University \\ of Oklahoma, ${ }^{2}$ Oklahoma Foundation \\ for Digestive Research, Oklahoma \\ City, Oklahoma, USA
}

\begin{abstract}
Nonsteroidal anti-inflammatory drugs (NSAIDs) are widely prescribed for reduction of pain and inflammation, particularly in the setting of rheumatologic disorders. While effective, they are associated with risks, including nephrotoxicity, gastrointestinal inflammation, peptic ulcer disease, and worsened cardiovascular outcomes. After development of cyclo-oxygenase 2 inhibitors to minimize gastrointestinal complications, early use revealed increased cardiovascular event rate risk, and retrospective analysis of traditional NSAIDs revealed similar concerns, with the exception of naproxen. PN400 is a fixed-dose combination formulation designed to provide sequential delivery of a nonenteric-coated, immediate-release esomeprazole $20 \mathrm{mg}$ mantle followed by an enteric-coated naproxen $500 \mathrm{mg}$ core. This review summarizes the pharmacokinetics, benefits, safety, and tolerability of PN400. Phase I trials demonstrated pharmacokinetics consistent with its formulation, and at different esomeprazole combination doses, PN400 containing esomeprazole $20 \mathrm{mg}$ was the lowest dose that still resulted in substantial sustained increases of gastric $\mathrm{pH}>4$. In two Phase III trials (Study 301 and Study 302), PN400 resulted in a significant reduction in gastric ulcers relative to enteric-coated naproxen (4.1\% to $23.1 \%$ in Study 301, $7.1 \%$ to $24.3 \%$ in Study 302). Discontinuation due to NSAID-associated upper gastrointestinal adverse events or duodenal ulcers was significantly less in PN400 patients ( $3.2 \%$ to $12 \%, P<0.001$, in Study $301 ; 4.8 \%$ to $11.9 \%, P=0.009$, in Study 302). Two subjective patient indices were utilized to assess tolerability, ie, the Severity of Dyspepsia Assessment (SODA) and Overall Treatment Evaluation of Dyspepsia (OTE-DP). Patients with PN400 had significantly better upper gastrointestinal tolerability compared with those treated with enteric-coated naproxen in terms of SODA scores, proportion of heartburnfree patients, and OTE-DP response. While no formal recommendations are available at this time for use of this new combination medication, it will likely become an important treatment option with application for many patients.
\end{abstract}

Keywords: esomeprazole, naproxen, combination, gastrointestinal tolerability

\section{Introduction}

Nonsteroidal anti-inflammatory drugs (NSAIDs) are among the most commonly prescribed medications worldwide. Their indications for reduction of pain, inflammation, and fever extend to a wide variety of musculoskeletal and inflammatory disorders, including rheumatoid arthritis and osteoarthritis. Despite their therapeutic effectiveness, NSAIDs are associated with risks, including nephrotoxicity, worsened cardiovascular outcomes, gastrointestinal inflammation, and peptic ulcer disease, among others. As our understanding of the mechanisms and etiologies of NSAIDinduced gastrointestinal injury has grown, new therapies have been developed to minimize that toxicity. In development and evaluation of these new therapies, greater
Correspondence: David Neil Roberts University of Oklahoma Digestive Diseases Section, 920 Stanton L Young Blvd, WP 1345, Oklahoma City,

OK 73104, USA

Tel +l 405 27I 5428

Fax +l $40527 \mid 5803$

Email david-roberts@ouhsc.edu
Drug, Healthcare and Patient Safety 20I I:3 I-8

(C) 20II Roberts and Miner, publisher and licensee Dove Medical Press Ltd.This is an Open Access article which permits unrestricted noncommercial use, provided the original work is properly cited.
Dovepress

DOI: 10.21 47/DHPS.S7329

\section{I} ' 
cardiovascular toxicity has been appreciable, evident for both traditional NSAIDs and selective inhibitors. The newest challenge has been seeking a balance with greatest benefit and least harm, often requiring a patient-individualized approach for these therapies.

Upper gastrointestinal complications are the most common risks of NSAID use. Resultant symptoms can include abdominal pain, diarrhea, dyspepsia, and gastrointestinal bleeding. Mucosal injury is mediated through a number of pathways, including an inhibition of cell proliferation, increase in apoptosis, inhibition of angiogenesis, creation of reactive oxygen species, loss of cytoskeletal control of tight junctions, increase in gastrointestinal permeability, and injury to blood vessels by an increase in adhesion molecules and injury to endothelial cells. A major source of NSAID injury is nonselective inhibition of the cyclo-oxygenase (COX) enzyme with resultant decreased production of prostaglandins. COX is the rate-limiting enzyme in the production of prostaglandins from arachidonic acid. Two isoforms have been identified, ie, COX-1 which is expressed constitutively and involved heavily in gastric mucosal integrity and the mucus barrier, and COX-2 which is inducible and found in areas of inflammation. Prostaglandin E2 (PGE2) induces protection by stimulating release of bicarbonate and mucus. The bicarbonate stimulatory effect of PGE2 is mediated by endogenous prostaglandin (EP)-1 receptors coupled with $\mathrm{Ca}^{2+}$ along the gastric mucosa, and EP-3 and EP-4 coupled intracellularly with $\mathrm{Ca}^{2+}$ and cyclic AMP in the duodenal mucosa., As such, prostaglandin analogs, misoprostol, H2-receptor antagonists (H2RA), and proton pump inhibitors have been evaluated in randomized controlled trials for the prevention of chronic NSAID-induced upper gastrointestinal toxicity. In a meta-analysis of these trials, misoprostol significantly reduced the risk of endoscopically visualized ulcers with a dose-response relationship in prevention of gastric ulcers at $400 \mu \mathrm{g}$ and $800 \mu \mathrm{g}$ daily (relative risk [RR] 0.17 and 0.39 , respectively, $P=0.0055)$. Misoprostol resulted in a reduction in risk of ulcer complications also, but resulted in diarrhea at all doses. Standard doses of $\mathrm{H} 2 \mathrm{RAs}$ reduced the risk of endoscopic duodenal ulcer (RR 0.36; 95\% confidence interval [CI] 0.18-0.74) but not gastric ulcers (RR 0.73; 95\% CI 0.50-1.08). Double-dose H2RAs and proton pump inhibitors were effective at reduction of endoscopic duodenal and gastric ulcers (RR 0.44; 95\% CI 0.26-0.74 and RR 0.40; 95\% CI 0.32-0.51, respectively, for gastric ulcer). These agents were better tolerated than misoprostol. ${ }^{3}$

The effect of NSAIDs on gastric secretory physiology is incompletely understood. Twenty-four hour gastric $\mathrm{pH}$ stud- ies have shown a lower mean 24-hour $\mathrm{pH}$. There are several possible explanations for this observation, including stimulation of gastric acid secretion. In a study of gastric acid secretory function, 24 patients were evaluated after one week of naproxen $500 \mathrm{mg}$ twice daily. Pentagastrin stimulation did not change maximum acid secretion. However, the gastric $\mathrm{pH}$ was lower in the basal acid secretion period, with no change in the number of $\mathrm{mEq}$ of acid secreted per hour. The basal total volume was decreased, suggesting that the reason the $\mathrm{pH}$ was lower is secondary to a naproxen-induced decrease in the nonacid fluid volume. ${ }^{4}$ Suppression of the acid $\mathrm{mEq}$ would, therefore, result in a re-established normal gastric $\mathrm{pH}$. The gastroprotective effects of proton pump inhibitors, working through decreasing acid secretion by inhibition of the $\mathrm{H}^{+}-\mathrm{K}^{+}$ATPase of the parietal cell, are more potent than other acid suppression classes. In addition to acid suppression, proton pump inhibitors have been noted to reduce oxidative stress by the induction of heme oxygenase- 1.5 Proton pump inhibitors have been shown to increase the strength of the gastric mucus barrier significantly ${ }^{6,7}$ and to inhibit neutrophil-derived oxygen free radical species., ${ }^{8,9}$ In a large-scale randomized comparison of twice-daily esomeprazole $20 \mathrm{mg}$ and $40 \mathrm{mg}$ with twice-daily ranitidine $150 \mathrm{mg}$ in Helicobacter pylorinegative patients with documented NSAID ulcers continuing to take NSAIDs, ulcer healing was seen in $85.7 \%, 84.8 \%$, and $76.3 \%$, respectively, at eight weeks. ${ }^{10}$ Another study evaluated 350 patients with NSAID-related gastric ulcers who continued to use NSAIDs, and $73 \%$ of patients given lansoprazole $30 \mathrm{mg}$ daily had ulcer healing at eight weeks compared with $69 \%$ of patients on lansoprazole $15 \mathrm{mg}$ daily and $53 \%$ of patients on ranitidine $150 \mathrm{mg}$ twice daily. ${ }^{11}$

Prevention of gastroduodenal ulcer recurrence with esomeprazole has been evaluated in high-risk patients with a history of peptic ulcer disease, aged over 60 years, and taking nonselective NSAIDs and COX-2 inhibitors. This was performed as two similar, double-blind, placebocontrolled, randomized, multicenter studies in the US and internationally. In the US study, the proportion of patients who developed ulcers over six months was $20.4 \%$ with placebo, $5.3 \%$ with esomeprazole $20 \mathrm{mg}$ daily, and $4.7 \%$ with esomeprazole $40 \mathrm{mg}$ daily. In the international study, peptic ulcer disease recurrence was seen in $12.3 \%$ on placebo, $5.2 \%$ on esomeprazole $20 \mathrm{mg}$, and $4.4 \%$ on esomeprazole $40 \mathrm{mg}$. Esomeprazole was effective in preventing ulcers in both the nonselective NSAID and COX-2 inhibitor groups. Of note, this study, like similar ones, demonstrates that esomeprazole $20 \mathrm{mg}$ daily is an effective dose for ulcer prevention in longterm NSAID users. ${ }^{12}$ 


\section{Risk of peptic ulcer disease}

In the framework of discussing the risk of peptic ulcer disease and its complications attributed to NSAIDs, a number of factors that alter the degree of risk are important. A history of previous peptic ulcer disease, presence of $H$. pylori, smoking, alcoholism, increased age, and concomitant use of corticosteroids, aspirin, other NSAIDs, and warfarin, are all important and increase the risk of peptic ulcer disease. While H. pylori is the cause of the majority of gastric and duodenal ulcers, aspirin and NSAIDs continue to be a common source, accounting for approximately $15 \%$ of duodenal ulcers and $26 \%$ of gastric ulcers. ${ }^{13}$ In an endoscopic study of chronic diclofenac users with rheumatoid arthritis or osteoarthritis, $24 \%$ of patients had gastric or duodenal ulcers. ${ }^{14}$ Regular NSAID usage occurs in $11 \%$ of the US population, which increases the odds of gastrointestinal bleeding five- to six-fold compared with those not taking NSAIDs. ${ }^{15,16}$ Some $1 \%-4 \%$ of NSAID users have serious ulcer-related complications every year. ${ }^{17}$ In many cases, life-threatening complications may be the first manifestation of peptic ulcer disease, as seen in a study of 235 patients, of whom $58 \%$ had previously been without symptoms. ${ }^{18}$ There is evidence that the individual NSAID may correlate with the risk of bleeding. This was seen in a case-control study of 2777 patients, with the highest risk of bleeding seen for ketorolac. ${ }^{19}$

Gastrointestinal safety and tolerability studies demonstrate that COX-2 inhibitors provide a significant risk reduction of gastroduodenal ulcers, ulcer complications, and gastrointestinal symptoms compared with traditional nonselective NSAIDs. The benefit of the COX-2 relative risk of ulcers (0.26) and ulcer complications (0.39) was significantly reduced when taken concomitantly with aspirin. ${ }^{20}$ The disrupted benefit of COX-2 by aspirin is felt to be the reason that no significant difference was seen in CLASS (the Celecoxib Long-Term Arthritis Safety Study) with regard to the incidence of ulcer complications between celecoxib and the nonselective NSAID group. ${ }^{21}$ This was better delineated in TARGET (the Therapeutic Arthritis Research and Gastrointestinal Event Trial) in which age and low-dose aspirin groups were stratified. The significantly reduced incidence of ulcer complications in the lumiracoxib group compared with nonselective NSAIDs was negated in the low-dose aspirin study arm. ${ }^{22}$

\section{Risk of cardiovascular events}

Despite the reduced gastrointestinal toxicity and improved safety profile of the COX-2 inhibitors, the increased cardiovascular risk has created appropriate hesitation in the use of these agents. During a study comparing rofecoxib with naproxen, the incidence of acute myocardial infarction was four times higher in the COX-2 inhibitor group. ${ }^{23}$ Cardiovascular safety was next analyzed by deriving from data in the two long-term studies of colon polyp prevention with rofecoxib, ie, the APPROVE (Adenomatous Polyp Prevention on Vioxx) study and celecoxib, ie, the APC (Adenoma Prevention with Celecoxib) study. In the APPROVE study, 18-month interim data indicated that patients receiving rofecoxib $25 \mathrm{mg}$ daily had twice the risk of serious cardiovascular events compared with patients receiving placebo. ${ }^{24}$ In the APC study, interim data at 33 months showed an occurrence of serious cardiovascular events significantly higher at the very high dose of $400 \mathrm{mg}$ twice daily only. ${ }^{25}$

Thereafter, traditional, nonselective NSAIDs were analyzed to examine for additional cardiovascular safety concerns. With the exception of full-dose naproxen (1000 mg a day), they also carry an increased cardiothrombotic risk. In a meta-analysis evaluating the risk of COX-2 inhibitors and nonselective NSAIDs, Kearney et al found that both groups of medications increased the risk of serious cardiovascular events more than placebo and naproxen. COX-2 inhibitors had a $42 \%$ relative increase in the incidence of serious vascular events compared with placebo, with no significant heterogeneity between the different selective COX- 2 inhibitors, attributed mainly to an increased risk of myocardial infarction. Overall, the incidence of serious vascular events was similar between a selective COX-2 inhibitor and any traditional NSAID. Compared with placebo, the summary rate ratio for vascular events was 0.92 for naproxen, 1.51 for ibuprofen, and 1.63 for diclofenac. ${ }^{26}$

\section{Enteric-coated naproxen + esomeprazole combination}

In light of the cardiovascular concerns, use of naproxen for chronic rheumatologic disorders will likely become more prevalent. Because proton pump inhibitor therapy has been shown to be an efficacious approach in reducing NSAIDassociated gastrointestinal injury, its use is recommended as a current prevention strategy. Despite this, gastroprotective treatments are underprescribed by providers, prescribed at suboptimal doses, or suffer from patient nonadherence resulting in worsened clinical outcomes. ${ }^{27} \mathrm{PN} 400$ is a fixed-dose combination formulation designed to provide sequential delivery of a nonenteric-coated, immediate-release esomeprazole $20 \mathrm{mg}$ mantle followed by an enteric-coated naproxen $500 \mathrm{mg}$ core. 
A crossover, open-label, single-center Phase I study comprising four treatment periods was performed in which patients were randomized into one of four treatment sequences for nine days, with a washout period of $\geq 14$ days between treatments. Patients were healthy adults aged $18-55$ years without prior history of peptic ulcer disease and who had tested negative for $H$. pylori. Twenty-eight patients were randomized and included in the safety, pharmacokinetics, and intention-to-treat populations. Twenty-seven patients completed the study, and 25 were included in the per-protocol population. Enteric-coated naproxen $500 \mathrm{mg}$ coupled with immediate-release esomeprazole $30 \mathrm{mg}, 20 \mathrm{mg}$, and $10 \mathrm{mg}$ (PN400/E30, PN400/E20, and PN400/E10, respectively) twice daily comprised three of the treatments. The fourth treatment was nonenteric-coated naproxen $500 \mathrm{mg}$ twice daily and esomeprazole $20 \mathrm{mg}$ daily. The study medication was administered 60 minutes prior to meals in the morning and evening. On day 9, esomeprazole had a corresponding dose-related effect on gastric $\mathrm{pH}$ beyond the influence of food intake. After administration of morning PN400 doses, rapid, dose-related increases in $\mathrm{pH}$ were seen approximately one hour earlier than food-induced increases in $\mathrm{pH}$. On the contrary, a delay in increased $\mathrm{pH}$ was seen following morning administration of naproxen and esomeprazole $20 \mathrm{mg}$. After nine days of treatment, PN400/E30 and PN400/E20 had similar effects on gastric $\mathrm{pH}$ resulting in a slower return to low $\mathrm{pH}$ after food consumption compared with PN400/E10 and naproxen and esomeprazole $20 \mathrm{mg}$. While initial doses of esomeprazole had only minimal effect on gastric $\mathrm{pH}$ beyond the effect of food intake, treatment differences emerged after the second dose.

Pharmacodynamic studies demonstrated several interesting features. On day 1 , measurable plasma concentrations of esomeprazole were obtained rapidly with all three PN400 treatments at 10 minutes after the morning dose and 20-30 minutes after the evening dose. It was rapidly eliminated from plasma in the majority of subjects by 6-8 hours after the dose from all three PN400 treatments. Regarding naproxen, plasma concentrations on day 1 were first detected at approximately two hours following PN400 administration for all three treatment groups. On day 9 , premorning dose samples showed measurable esomeprazole concentrations in 19 patients at the PN400/E30 dose, 13 patients at the PN400/E20 dose, and six patients at the PN400/E10 dose. Analyses after the morning and evening doses showed that for the PN400 treatments with higher esomeprazole doses (PN400/E30 and PN400/E20), plasma concentrations of esomeprazole were measurable at earlier time points after dosing in a large number of patients and for a longer period of time compared with day 1 . Samples before the morning dose showed measurable naproxen concentrations throughout the various sampling times. On day 9, all subjects had measurable naproxen concentrations before the morning dose. Following the morning and evening doses on days 1 and 9, plasma esomeprazole concentrations increased with the esomeprazole dose for all three PN400 treatments. Maximum plasma concentrations for naproxen occurred after approximately 3-4 hours and was higher after the morning dose than after the maximal evening dose at 10-14 hours on day 1 and day 9 for all three PN400 treatments. Following treatment with esomeprazole $20 \mathrm{mg}$ and nonenteric-coated naproxen, naproxen was quickly absorbed, with peak plasma concentrations observed at 1.5 hours postdose on days 1 and 9 in the majority of subjects, and were measurable for longer than concentrations following PN400 administration. This plasma esomeprazole profile was consistent with a delayedrelease formulation. Mean plasma profiles of naproxen were comparable following the three PN400 treatments which is consistent with the delayed-release formulation. Higher mean naproxen plasma concentration levels observed at the end of a 24-hour daily interval are the result of a delayed absorption of naproxen from PN400. Mean plasma concentrations of naproxen were much higher on day 9 than day 1 . Following treatment with naproxen and esomeprazole $20 \mathrm{mg}$ on day 1 , plasma naproxen concentrations were measurable in all subjects at the 10-minute postdose sample time and for up to 24 hours thereafter, which is consistent with a nonentericcoated naproxen formulation. After morning and evening doses on days 1 and 9, mean plasma naproxen concentrations were higher and occurred earlier following naproxen with enteric-coated esomperazole $20 \mathrm{mg}$ than any of the PN400 treatments.

The pharmacokinetic parameters of PN400 following administration of the morning and evening doses on days 1 and 9 are summarized in the Table 1. Peak esomeprazole plasma concentrations occurred approximately 0.5 hours after the morning dose, and between 1.0-1.5 hours after the evening dose on days 1 and 9 for all three PN400 treatments. Peak naproxen plasma concentrations occurred approximately 3-4 hours after the morning dose, and between 10-14 hours after the evening dose on days 1 and nine for all three PN400 formulations. The delay in naproxen absorption is consistent with the enteric-coated naproxen in PN400. Peak plasma esomeprazole concentrations and areas under the concentration-time curve were higher following the morning dose than the evening dose on days 1 and 9 for all three 
Table I Pharmacokinetics of naproxen + esomeprazole combinations*

\begin{tabular}{|c|c|c|c|c|c|c|}
\hline \multirow{2}{*}{$\begin{array}{l}\text { Treatment, day } \\
\text { and dose }\end{array}$} & \multicolumn{3}{|l|}{ Esomeprazole } & \multicolumn{3}{|l|}{ Naproxen } \\
\hline & $C_{\max }(n g / m L)$ & $\mathbf{t}_{\max }(\mathrm{h})$ & AUC $(\mathrm{h} * \mathrm{ng} / \mathrm{mL})$ & $C_{\max }(n g / m L)$ & $\mathbf{t}_{\max }(\mathrm{h})$ & AUC $\left(h^{*} n g / m L\right)$ \\
\hline \multicolumn{7}{|c|}{ PN 400/E30 (n= 28) } \\
\hline Day I, morning & $487(82)$ & $0.50(0.33-1.50)$ & $591(108)$ & $48.1(53)$ & $4.00(2.00-10.00)$ & $259(56)$ \\
\hline Day I, evening & $187(132)$ & $1.50(0.33-4.00)$ & $388(137)$ & $68.9(28)$ & $14.00(0.50-14.00)$ & $47 \mid(30)$ \\
\hline \multicolumn{7}{|c|}{ PN 400/E20 (n= 28) } \\
\hline Day I, morning & $292(77)$ & $0.50(0.20-1.50)$ & $350(113)$ & $44.4(68)$ & $4.00(2.00-10.00)$ & $231(70)$ \\
\hline Day I, evening & $96.6(104)$ & $1.49(0.33-3.00)$ & $206(|4|)$ & $71.5(26)$ & $\mid 4.00(0.00-14.00)$ & $450(33)$ \\
\hline \multicolumn{7}{|c|}{ PN 400/EI0 (n=27) } \\
\hline Day I, morning & I38 (7I) & $0.33(0.17-3.10)$ & I48 (III) & $57.0(31)$ & $4.00(2.00-10.00)$ & $310(35)$ \\
\hline Day I, evening & $35.3(84)$ & $1.50(0.33-3.00)$ & $85.7 \neq(179)$ & $68.6(26)$ & $10.00(0.00-14.00)$ & $508(29)$ \\
\hline \multicolumn{7}{|c|}{ Naproxen + enteric-coated } \\
\hline \multicolumn{7}{|l|}{ E20 $(n=28)$} \\
\hline Day I, morning & $282(66)$ & $1.50(1.00-16.00)$ & $540 \neq(60)$ & $65.5(25)$ & $1.50(0.75-6.00)$ & $409(16)$ \\
\hline Day I, evening & & & & $81.5(14)$ & $1.50(0.50-2.50)$ & $685(10)$ \\
\hline \multicolumn{7}{|c|}{ PN 400/E30 $(n=28)$} \\
\hline Day 9, morning & 1584 (39) & $0.50(0.17-1.50)$ & $2779(45)$ & $80.9(23)$ & $3.00(0.00-8.00)$ & $603(2 I)$ \\
\hline Day 9 , evening & $810(59)$ & $1.00(0.33-8.00)$ & $2066(53)$ & $76.2(23)$ & $10.40(0.00-14.00)$ & $648(20)$ \\
\hline \multicolumn{7}{|c|}{ PN 400/E20 ( $n=27)$} \\
\hline Day 9, morning & $715(52)$ & $0.50(0.17-1.50)$ & $1216(69)$ & $86.2(22)$ & $3.00(0.00-8.05)$ & $607(19)$ \\
\hline Day 9, evening & $428(73)$ & $0.75(0.33-3.00)$ & $919(84)$ & $76.8(18)$ & $10.00(0.00-14.00)$ & $678(16)$ \\
\hline \multicolumn{7}{|c|}{ PN 400/EIO (n= 27) } \\
\hline Day 9 , morning & $278(57)$ & $0.33(0.17-1.00)$ & $368(89)$ & $87.1(21)$ & $2.50(0.00-8.00)$ & $637(17)$ \\
\hline Day 9, evening & $976(136)$ & $1.00(0.33-2.00)$ & $223 \neq(134)$ & $78.6(17)$ & $14.00(\mid .50-14.00)$ & $672(19)$ \\
\hline \multicolumn{7}{|c|}{ Naproxen + enteric-coated } \\
\hline Day 9, morning & $435(48)$ & $1.50(1.00-14.00)$ & $1046(54)$ & $90.0(19)$ & $1.50(0.50-4.00)$ & $617(12)$ \\
\hline Day 9 , evening & & & & $86.5(13)$ & $1.50(0.75-4.00)$ & $769(10)$ \\
\hline
\end{tabular}

Notes: Values are mean (\% coefficient of variation) for $C_{\max }$ and $A U C$ and medial (range) for $t_{\max } ; * A \cup C_{0-10, A M}$ or $A U C_{0-14, P M} \neq n=26 ; * R e f e r e n c e ~ 28$.

Abbreviations: $\mathrm{PK}$, pharmacokinetic; $\mathrm{C}_{\text {max }}$, peak plasma concentration; $\mathrm{t}_{\max }$, time to peak plasma concentration; $\mathrm{AUC}$, area under the plasma concentration vs time curve.

PN400 treatments and were dose-dependent. Esomeprazole concentrations were higher on day 9 than day 1 for each PN400 treatment. Peak plasma naproxen concentrations and areas under the concentration-time curve after the morning and evening doses were comparable among all three PN400 treatments, expected with the same dose of naproxen in each formulation..$^{28}$

Two Phase III studies (Study 301, $\mathrm{n}=438$ and Study $302, n=423$ ) compared the upper gastrointestinal efficacy and safety of PN400 with enteric-coated naproxen alone in patients at risk of NSAID-associated ulcers. Evaluations were randomized, double-blind, controlled, parallel-group, multicenter studies with $H$. pylori-negative patients with osteoarthritis, rheumatoid arthritis, or another condition requiring chronic NSAIDs without baseline peptic ulcer disease aged $\geq 50$ years or $18-49$ years with a history of peptic ulcer disease in the past five years. Treatment was twice daily in both arms, and tolerability, safety, and endoscopic analysis were performed at baseline, at 30 and 60 days, and at six-monthly intervals thereafter. The primary endpoint of the studies was cumulative incidence of gastric ulcers, defined as a mucosal break $\geq 3 \mathrm{~mm}$ in depth throughout the six months of therapy. The analysis was designed for stratification by low-dose aspirin usage ( $\leq 325 \mathrm{mg} /$ day). Secondary endpoints included cumulative incidence of duodenal ulcers, discontinuation of the medication as a result of any adverse event, and incidence of prespecified NSAID-associated upper gastrointestinal adverse events and/or duodenal ulcers. Secondary endpoints also included a number of patient reported outcomes which will be discussed later in the tolerability section.

In Study 301, the cumulative incidence of gastric ulcers in the PN400 arm was $4.1 \%$ compared with $23.1 \%$ in the enteric-coated naproxen arm. Similarly, in Study 302, a significant reduction of gastric ulcers was seen in $7.1 \%$ of PN400 patients relative to $24.3 \%$ of naproxen patients. The incidence of discontinuations from the study secondary to NSAID-associated upper gastrointestinal adverse events or duodenal ulcers was significantly less in PN400 patients. In Study 301, this included 3.2\% compared with $12 \%$ in the enteric-coated naproxen arm $(P<0.001)$. In Study 302, $4.8 \%$ of PN400 patients discontinued for these reasons, along 
with $11.9 \%$ in the naproxen group $(P=0.009)$. Importantly, evaluation of patients on low-dose aspirin also showed a protective benefit in the PN400 group, with a lower combined incidence of gastric ulcers $(3.0 \%)$ versus enteric-coated naproxen $(28.4 \%){ }^{27}$

\section{Safety and tolerability}

In the Phase I study, clinical adverse events (at least one) were reported in half of the patients in the PN400/E30 and PN400/E20 treatment groups (14/28). A third of patients on the PN400/E10 treatment were affected. Interestingly, only $8 / 28$ patients $(29 \%)$ reported at least adverse event on the traditional naproxen with once-daily esomeprazole $20 \mathrm{mg}$ treatment. In the further studied PN400/E20 formulation, 29\% had adverse events in the form of gastrointestinal disorders, manifested as diarrhea (14\%), abdominal distension (7\%), and dyspepsia (7\%). Of note, in this limited evaluation, upper abdominal pain and gastroenteritis were not demonstrated for the PN400/E20 treatment but were seen for the formulations with higher and lower esomeprazole doses (PN400/E30 and PN400/E10). Metabolism and nutrition disorders (18\%), iron deficiency (18\%), and headaches (4\%) were also seen for the PN400/E20 treatment. No serious adverse events were reported, and no patient withdrew from the study due to adverse events. ${ }^{28}$

During the Phase III studies (301 and 302), safety was assessed by the incidence of adverse events, treatmentrelated adverse events, and serious adverse events. These were assessed and captured throughout the trial by study personnel questioning, patient reporting of symptoms, physical examinations, laboratory assessments, and endoscopic findings. Clinical laboratory testing consisted of a hepatic function panel, blood urea nitrogen, creatinine, and complete blood count at screening and/or baseline and at months 1,3 , and 6 . In the safety population, the overall incidence of treatment-emergent adverse events was similar between treatment groups in both studies (78\% versus $81.5 \%$ for Study $301 ; 76.2 \%$ versus $82.9 \%$ for Study 302 ). The most common treatment-emergent adverse events were gastrointestinal disorders seen in the form of patient-reported symptoms and/or endoscopic findings, which were more frequent in the enteric-coated naproxen group than in the PN400 groups.

The incidence of adverse events related to the study medication was higher with enteric-coated naproxen than PN400 in both studies. It is important to recognize that while a significant number of patients in the PN400 group had adverse events, many of these were endoscopic findings and did not correlate with patient symptoms. Adverse events were comparable between the study drug groups in the number of patients having an event with infections and infestations (16\%-19\%). In Study 302, the percentage of patients having an event related to nervous system disorders (7.6\%), musculoskeletal disorders (9.5\%), and respiratory, thoracic, and mediastinal disorders (4.8\%) were identical between PN400 and enteric-coated naproxen. In Study 301, slightly fewer patients had problems in the enteric-coated naproxen group compared with the PN400 group in musculoskeletal disorders (6\% versus $8.3 \%)$ and nervous system disorders (4.2\% versus $9.6 \%)$, likely related to headache.

In the category of gastrointestinal disorder-related adverse events, far fewer patients in the PN400 group had problems relative to enteric-coated naproxen $(61.5 \%$ versus $75 \%$ for Study 301; 60.5\% versus $71.9 \%$ for Study 302 ). Far less esophagitis, erosive esophagitis, duodenitis, erosive duodenitis, erosive gastritis, upper abdominal pain, and dyspepsia were seen in the PN400 populations. Gastritis, diarrhea, nausea, and abdominal distension were variable between the studies, but fairly comparable overall, with a trend of benefit toward PN400. In Study 301, serious adverse events related to study treatment were duodenal ulcer hemorrhage $(n=1)$ and noncardiac chest pain $(n=1)$, in both the enteric-coated naproxen groups. No serious treatmentrelated adverse events were present in Study 302, nor were there any deaths in either study.

With regard to tolerability, two subjective patient-recorded indices were utilized to assess upper gastrointestinal symptoms. The Severity of Dyspepsia Assessment (SODA) and Overall Treatment Evaluation of Dyspepsia (OTE-DP) scoring systems have been utilized in previous clinical trials..$^{29,30}$ The SODA questionnaire was completed at baseline and at months 1,3 , and 6, and comprised 17 questions measuring three categories of dyspepsia, ie, pain intensity, symptoms not related to pain, and satisfaction with dyspepsia-related health. The OTE-DP questionnaire is derived from the Global Ratings of Change questionnaire and was utilized at month 6 or at withdrawal. Patients with PN400 had significantly better upper gastrointestinal tolerability compared with those treated with enteric-coated naproxen in terms of SODA scores, proportion of heartburnfree patients, and OTE-DP response. The change from baseline to six months in SODA scores in all three domains were dramatically in favor of PN400. In the pain intensity category, an average drop of 6.61 points compared with 0.16 with enteric-coated naproxen $(P<0.001)$ in Study 301 . 
In Study 302, a drop of 2.84 over 0.08 was seen $(P=0.004)$. In nonpain symptoms, PN400 was superior $(-2.18$ versus -0.47 in Study $301 ;-1.11$ versus 0.11 for Study 302 ). Overall improvement of satisfaction scores increased by 3.36 and 1.88 in the PN400 groups compared with 0.87 and 0.47 , respectively, for Study 301 and Study 302. In the OTE-DP assessment, patients in both Study 301 and Study 302 noted better symptoms relative to baseline with PN400, and worse symptoms with enteric-coated naproxen. ${ }^{27}$

\section{Implications for future work and improved patient care}

Gastroprotective strategies, particularly with proton pump inhibitors, have been shown to prevent and reduce NSAIDassociated ulcers, ulcer complications, and bleeding, as well as NSAID-related heartburn and acid regurgitation. As the population ages, and the indications and usage of NSAIDs continue to climb, we must seek improved ways to be proactive in prevention of gastrointestinal toxicity. Adherence to gastroprotective agents by patients on chronic NSAIDs was less than $40 \%$ in two retrospective studies. ${ }^{31,32}$ For this reason, the need for improved physician and patient education, strategies to improve patient compliance, and the use of a fixed-dose combination of NSAID and gastroprotective agent to target issues with compliance will all be key aspects for improved patient outcomes in the near future. No data exist to indicate that a proton pump inhibitor will reduce the complications of NSAIDs related to renal insufficiency, cardiovascular risk, and enteric or colonic complications, so more investigation into the risk and incidence of these complications is important. Some recent data raise concern that, while significant upper gastrointestinal toxicity from NSAIDs has diminished, lower gastrointestinal toxicity and events are on the rise. This has resulted in a calling for more comprehensive gastrointestinal endpoints, like the Clinically Significant Upper or Lower GI Events (CSULGIEs), as we examine these agents. ${ }^{33,34}$ It is certain that further work in traditional NSAID/gastroprotectant combinations and different selective inhibitor agents will occur.

Other combination therapies include diclofenac + misoprostol and ibuprofen + famotidine, which have also demonstrated a reduction in the endoscopic incidence of gastric ulcers. A subject of important debate is whether measurement of endoscopic ulcers as a primary endpoint is clinically important and valid. At this time, the evidence suggests that it is a reasonable and acceptable endpoint for clinical trials. While the data support the superiority of proton pump inhibitors over $\mathrm{H} 2$ blockers and prostaglandin analogs in the prevention of chronic NSAID-induced gastroduodenal ulcers, the only true determination of the relative efficacy would be a direct comparison study.

\section{Conclusion}

The choice of an anti-inflammatory agent and use of gastroprotection is an area of intense interest at this time, and requiring careful awareness of the individual patient's history and risk for cardiovascular, cerebrovascular, and gastrointestinal events. The complexity of weighing these risk factors and making appropriate treatment recommendations is in a period of flux. Current recommendations are that patients with cardiovascular risk on aspirin who require NSAIDs should be treated with naproxen and a proton pump inhibitor for gastroprotection. For those patients with a risk of gastrointestinal bleeding but without cardiovascular risk factors, a COX-2 inhibitor or a nonselective NSAID with a proton pump inhibitor is recommended..$^{35}$

While upper gastrointestinal events have been characterized and studied extensively, the incidence of lower gastrointestinal events and toxicity is poorly quantified. With an increase in lower gastrointestinal toxicity, study endpoints in the future will likely need to incorporate this to be able to counsel patients and select the appropriate agents. Further development of combination treatments will undoubtedly improve compliance and will reduce the risk of gastrointestinal complications in chronic NSAID users.

\section{Disclosure}

PBM, Jr, performed some of the studies referred to in this manuscript with grants from Pozen Inc and AstraZeneca. He has also served on medical advisory boards and acted as a consultant for Pozen Inc and AstraZeneca.

DNR has no disclosures to make.

\section{References}

1. Takeuchi K, Yahi K, Kato S, et al. Roles of prostaglandin E-receptor subtypes in gastric and duodenal bicarbonate secretion in rats. Gastroenterology. 1997;113(5):1553-1559.

2. Takeuchi K, Aihara E, Hayashi M, et al. Role of prostaglandin E receptor subtypes in gastroduodenal $\mathrm{HCO}_{3}$ secretion. Med Chem. 2005; 1(4):395-403

3. Rostom A, Dube C, Wells GA, et al. Prevention of NSAID-induced gastroduodenal ulcers. Cochrane Database Syst Rev. 2002;4: CD002296.

4. Rodriguez-Stanley S, Redinger N, Miner PB. Effect of naproxen on gastric acid secretion and gastric $\mathrm{pH}$. Aliment Pharmacol Ther. 2006;23(12):1719-1724.

5. Becker JC, Grosser N, Waltke C, et al. Beyond gastric acid reduction: Proton pump inhibitors induce heme oxygenase-1 in gastric and endothelial cells. Biochem Biophys Res Commun. 2006;345(3): 1014-1021. 
6. Blandizzi C, Gherardi G, Natale G, et al. Protective action of omeprazole against gastric mucosal injury induced by hemorrhagic shock in rats. Dig Dis Sci. 1994;39(10):2109-2017.

7. Blandizzi C, Gherardi G, Marveggio C, et al. Mechanisms of protection by omeprazole against experimental gastric mucosal damage in rats. Digestion. 1995;56(3):220-229.

8. Wandall JH. Effects of omeprazole on neutrophil chemotaxis, super oxide production, degranulation, and translocation of cytochrome b-245. Gut. 1992;33(5):617-621.

9. Suzuki M, Nakamura M, Mori M, et al. Lansoprazole inhibits oxygen-derived free radical production from neutrophils activated by Helicobacter pylori. J Clin Gastroenterol. 1995;20 Supp1 2:S93-S96.

10. Goldstein JL, Johanson JF, Hawkey CJ, et al. Clinical trial: Healing of NSAID-associated gastric ulcers in patients continuing NSAID therapy - a randomized study comparing ranitidine with esomeprazole. Aliment Pharmacol Ther. 2007;26(8):1101-1111.

11. Agrawal NM, Campbell DR, Safdi MA, et al. Superiority of lansoprazole vs ranitidine in healing nonsteroidal anti-inflammatory drug-associated gastric ulcers: Results of a double-blind, randomized, multicenter study. NSAID-Associated Gastric Ulcer Study Group. Arch Intern Med. 2000; 160(10):1455-1461.

12. Scheiman JM, Yeomans ND, Talley NJ, et al. Prevention of ulcers by esomeprazole in at-risk patients using non-selective NSAIDs and COX-2 inhibitors. Am J Gastroenterol. 2006;101(4):701-710.

13. Musumba C, Prichard DM, Pirmohamed M. Review article: Cellular and molecular mechanisms of NSAID-induced peptic ulcers. Aliment Pharmacol Ther. 2009;30(6):517-531.

14. Geis GS, Stead H, Wallemark CB, et al. Prevalence of mucosal lesions in the stomach and duodenum due to chronic use of NSAID in patients with rheumatoid arthritis or osteoarthritis, and interim report on prevention by misoprostol of diclofenac associated lesions. J Rheumatol. 1991;18 Suppl 28:11-14.

15. Kaufman D, Kelly JP, Rosenberg L, et al. Recent patterns of medication use in the ambulatory adult population of the United States: The Slone survey. JAMA. 2002;287(3):337-344.

16. Garcia Rodriguez LA, Hernandez-Diaz S. Relative risk of upper gastrointestinal complications among users of acetaminophen and nonsteroidal anti-inflammatory drugs. Epidemiology. 2001;12(5):570-576.

17. Wilcox C, Allison J, Benzuly K, et al. Consensus development conference on the use of nonsteroidal anti-inflammatory agents, including cyclooxygenase-2 enzyme inhibitors and aspirin. Clin Gastroenterol Hepatol. 2006;4(9):1082-1089.

18. Armstrong CP, Blower AL. Non-steroidal anti-inflammatory drugs and life threatening complications of peptic ulceration. Gut. 1987;28(5): $527-532$.

19. Lanas A, Garcia-Rodriguez LA, Arroyo MT, et al. Risk of upper gastrointestinal ulcer bleeding associated with selective cyclooxygenase-2 inhibitors, traditional non-aspirin non-steroidal anti-inflammatory drugs, aspirin, and combinations. Gut. 2006;55(12):1731-1738.

20. Rostom A, Muir K, Dube C, et al. Gastrointestinal safety of cyclooxygenase-2 inhibitors: A Cochrane Collaboration systematic review. Clin Gastroenterol Hepatol. 2007;5(7):818-828.

21. Silverstein FE, Faich G, Goldstein JL, et al. Gastrointestinal toxicity with celecoxib vs nonsteroidal anti-inflammatory drugs for osteoarthritis and rheumatoid arthritis: The CLASS study: A randomized controlled trial. Celecoxib long-term arthritis safety study. JAMA. 2000;284(10):1247-1255.

Drug, Healthcare and Patient Safety

\section{Publish your work in this journal}

Drug, Healthcare and Patient Safety is an international, peer-reviewed open-access journal exploring patient safety issues in the healthcare continuum from diagnostic and screening interventions through to treatment, drug therapy and surgery. The journal is characterized by the rapid reporting of reviews, original research, clinical, epidemiological and
22. Schnitzer TJ, Burmester GR, Mysler E, et al. Comparison of lumiracoxib with naproxen and ibuprofen in the Therapeutic Arthritis Research and Gastrointestinal Event Trial (TARGET), reduction in ulcer complications: Randomized controlled trial. Lancet. 2004;364(9435): 665-674.

23. Bombardier C, Laine L, Reicin A, et al. Comparison of upper gastrointestinal toxicity of rofecoxib and naproxen in patients with patients with rheumatoid arthritis. VIGOR Study Group. N Engl J Med. 2000; 343(20):1520-1528.

24. Bresalier RS, Sandler RS, Quan H, et al. Cardiovascular events associated with rofecoxib in a colorectal adenoma chemoprevention trial. N Engl J Med. 2005;352(11):1092-1102.

25. Solomon SD, McMurray JJ, Pfeffer MA, et al. Cardiovascular events associated with celecoxib in a clinical trial for colorectal adenoma prevention. N Engl J Med. 2005;352(11):1071-1080.

26. Kearney PM, Baigent C, Godwin J, et al. Do selective cyclooxygenase- 2 inhibitors and traditional non-steroidal anti-inflammatory drugs increase the risk of atherothrombosis? Meta-analysis of randomized trials. BMJ. 2006;332(7553):1302-1308.

27. Goldstein JL, Hochberg MC, Fort JG, et al. Clinical trial: The incidence of NSAID-associated endoscopic gastric ulcers in patients treated with PN 400 (naproxen plus esomeprazole magnesium) versus enteric-coated naproxen alone. Aliment Pharmacol Ther. 2010;32(3):401-413.

28. Miner PB, Plachetka JR, Orlemans E, Fort JG, Sostek MB. Clinical trial: Evaluation of gastric acid suppression with three doses of immediaterelease esomeprazole in the fixed-dose combination of PN 400 (naproxen/esomeprazole magnesium) compared with naproxen $500 \mathrm{mg}$ and enteric coated esomeprazole $20 \mathrm{mg}$ : A randomized, open-label, Phase I study in healthy volunteers. Aliment Pharmacol Ther. 2010; 32(2):414-424.

29. Rabeneck L. Measuring dyspepsia-related health in randomized trials: The Severity of Dyspepsia Assessment (SODA) and its use in treatment with NSAIDs and COX-2 specific inhibitors. Rheumatology. 2003;42 Suppl 3:32-39.

30. Jaeschke R, Singer J, Guyatt GH. Measurement of health status. Ascertaining the minimal clinically important difference. Control Clin Trials. 1989;10(4):407-415.

31. Goldstein JL, Howard KB, Walton SM, et al. Impact of adherence to concomitant gastroprotective therapy on nonsteroidal-related gastroduodenal ulcer complications. Clin Gastroenterol Hepatol. 2006;4(11): 1337-1345.

32. Sturkenboom MC, Burke TA, Tangelder MJ, et al. Adherence to proton pump inhibitors or H2-receptor antagonists during the use of non-steroidal anti-inflammatory drugs. Aliment Pharmacol Ther. 2003; 18(11-12):1137-1147.

33. Lanas A. A review of the gastrointestinal safety data - a gastroenterologist's perspective. Rheumatology. 2010;49 Supp1 2:3-10.

34. Chan FK, Cryer B, Goldstein JL, et al. A novel composite endpoint to evaluate the gastrointestinal (GI) effects of nonsteroidal antiinflammatory drugs through the entire GI tract. $J$ Rheumatol. 2009;37(1): 167-174.

35. Scheiman JM, Hindley CE. Strategies to optimize treatment with NSAIDs in patients at risk for gastrointestinal and cardiovascular adverse events. Clin Ther. 2010;32(4):667-677.

post-marketing surveillance studies, risk management, health literacy and educational programs across all areas of healthcare delivery. The manuscript management system is completely online and includes a very quick and fair peer-review system. Visit http://www.dovepress.com/ testimonials.php to read real quotes from published authors. 